\title{
Forschungsinfrastrukturen
}

\section{Modernes Forschungsdatenmanagement - ein Naturstoffprojekt als Fallstudie}

\author{
VANESSA KOCH, STEFAN BRÄSE \\ INSTITUT FÜR ORGANISCHE CHEMIE UND INSTITUT FÜR BIOLOGISCHE UND CHEMISCHE SYSTEME - IBCS-FMS, \\ KARLSRUHER INSTITUT FÜR TECHNOLOGIE (KIT), KARLSRUHE
}

DOI: $10.1007 / \mathrm{s} 12268-020-1316-3$

(C) Die Autoren 2020

Seit Ende 2014 werden am Karlsruher Institut für Technologie (KIT) synthetische Methoden zur Herstellung von Steroiden mit interessanten biologischen Eigenschaften (Cardenolide, Gorgosterol-Derivate) untersucht. Strukturell basieren Steroide (Abb. 1, Mitte) auf einem Ringsystem, das tetrazyklisch aufgebaut ist, und zeichnen sich durch ihre hohe Lipophilie aus. Sie übernehmen zahlreiche Funktionen im menschlichen Körper und in Pflanzen (Abb. 1). Steroide sind beispielsweise ein wichtiger Bestandteil von Zellmembranen (Cholesterin), beeinflussen als Sexualhormone (z. B. Testosteron, Estrogen) zahlreiche regulierende Prozesse in Zellen oder dienen als Gallensäure der Fettverdauung. Cardenolide oder Bufadienolide sind aufgrund ihrer Toxizität als Gifte bekannt und bilden auf diese Weise die Grundlage für Überlebensstrategien von Tieren und Pflanzen [1-3]. Steroide haben auch in der Medizin einen hohen Stellenwert und können beispielsweise als Entzündungshemmer (Cortison), Muskelrelaxans (Vecuronium) oder als Herzglykoside (Digitoxin, Klasse der Cardenolide) zur Behandlung von kongestiven Herzerkrankungen eingesetzt werden. Neben den bereits erwähnten natürlich vorkommenden Steroiden wurden auch zahlreiche synthetische Steroide mit interessanten medizinischen Anwendungen entwickelt. Darunter fällt beispielsweise die Antibabypille zur Empfängnisverhütung, deren Wirkung auf dem synthetischen Ethinylestradiol beruht.

Von besonderem Interesse für die Forscher am KIT ist Calotropin, das in der Vergangenheit aus verschiedenen Pflanzen der Unterfamilie der Seidenpflanzengewächse (z. B. Calotropis gigantea, Calotropis procera, Asclepias curassavica) [4-6] isoliert werden konnte. In Ergänzung zur Naturstoffisolation kann eine Semisynthese, die die Modifikation eines kommerziellen Steroidgrundgerüstes nutzt, einen schnellen und modularen Zugang zu den hochfunktionalisierten Steroiden ermöglichen. Durch den Zusammenschluss verschiedener Forschungsaktivitäten am KIT konnte eine Kombination aus Naturstoffisolation und-synthese einen wertvollen Beitrag zur Bereitstellung neuer Leitstrukturen leisten. Zur Realisierung der angestrebten Ziele sowie der Dokumentation des Forschungsfortschritts wurde eine über die letzten Jahre aufgebaute Infrastruktur genutzt, die aus drei Komponenten besteht: einem Molekülarchiv zur Substanzeinlagerung [7], einem elektronischen Laborjournal (electronic laboratory notebook, ELN) [8] und einem Repositorium [9] für Forschungsdaten. Das Zusammenwirken der einzelnen
Komponenten der Infrastruktur soll insbesondere bei langjährigen Projekten und vielfältigen Beiträgen zu Forschungsprojekten die weiterführende Nutzung der Ergebnisse ermöglichen und so ein effizientes Forschungs(daten)management gewährleisten.

\section{Das Molekülarchiv}

Das 2016 gegründete Molekülarchiv der Compound Platform des KIT (ComPlat) hat es sich zur Aufgabe gemacht, chemische Verbindungen zu katalogisieren, sachgerecht zu lagern und anderen Wissenschaftlern zur Verfügung zu stellen („Ein Molekülarchiv als akademisch integrierte Service-Einrichtung“, BIOspektrum, 2017 [7]). Das Molekülarchiv umfasst nicht nur Naturstoffe und biologisch aktive Substanzen, sondern auch deren synthetische Vorläufer, Liganden, Materialien

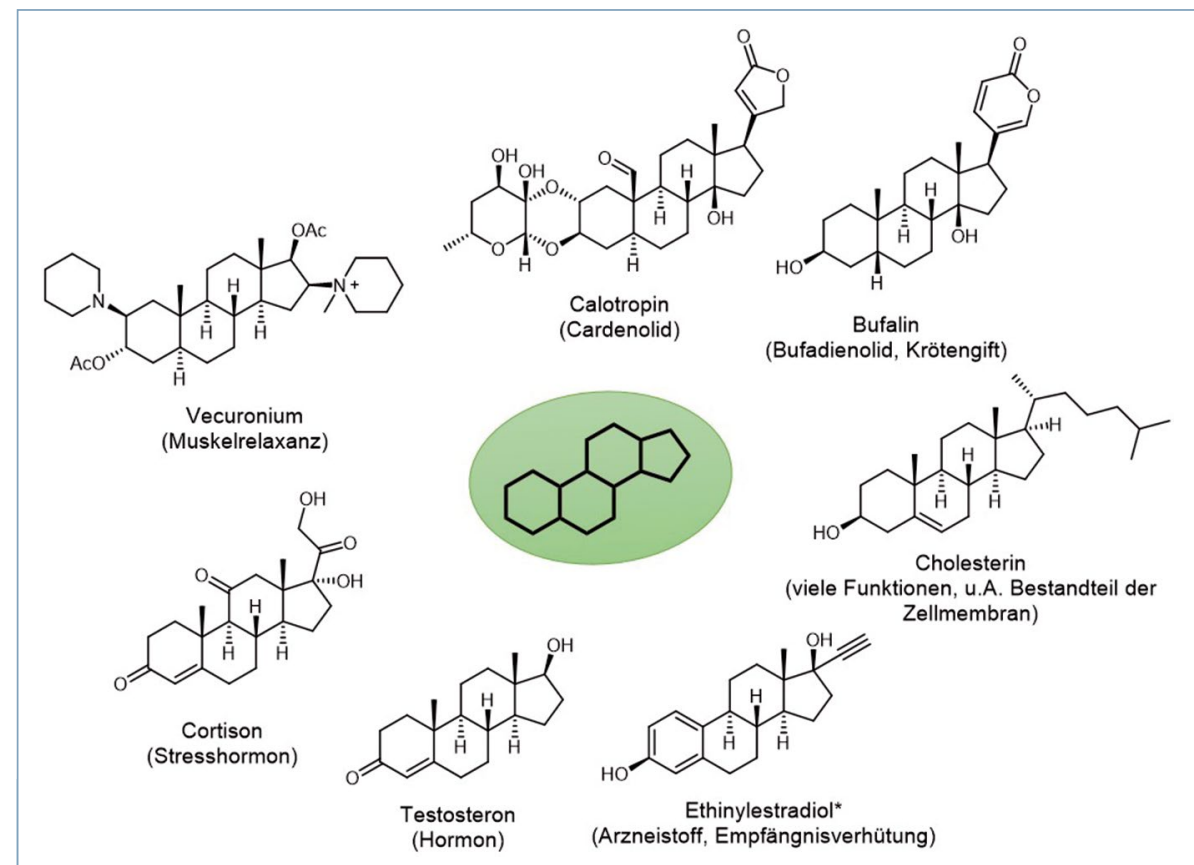

$\Delta$ Abb. 1: Übersicht über verschiedene natürlich vorkommende und synthetische Steroide (mit Stern markiert) sowie deren Funktion bzw. Anwendung in der Medizin. Calotropin stellt die Leitstruktur der Arbeiten des Arbeitskreises Bräse dar, fungiert unter anderem als Wnt-Inhibitor $[16,17]$ und kann aus diversen Seidenpflanzengewächsen isoliert werden. 
sowie weitere organische Substanzen. Neben der Katalogisierung der chemischen Verbindung, wird die Nachnutzung (z. B. in Form von toxikologischen oder anderen biologischen Tests) nach Absprache übernommen. Das Molekülarchiv wird von verschiedenen Gruppen genutzt, um Substanzen anderen Gruppen zugänglich zu machen. Es dient ebenfalls als Archiv für Substanzen von nicht mehr aktiv arbeitenden Arbeitsgruppenleitern. Die Arbeiten an dem Naturstoff Calotropin wurden durch eine Substanzspende von Dr. H. T. Andrew Cheung (University of Sydney, Australien) [10-12] an das Molekülarchiv initiiert. Nach der Archivierung und Evaluierung der erhaltenen Substanzen wurden erste biologische Tests durchgeführt (Abb. 2, Mitte), die zu einer weiteren Untersuchung der Substanzklasse führten. Parallel wurden zwei Wege verfolgt, um durch eine möglichst komplementäre Herangehensweise ein breites Spektrum der Calotropin-Derivate herstellen und testen zu können: Einerseits wurden durch eine Aufzucht der Calotropis-Pflanze in den botanischen Gärten des KIT Calotropin (Prof. Dr. Peter Nick, Botanisches Institut, KIT) und verwandte Strukturen nach literaturbekannten Protokollen isoliert (Abb. 2, links). Andererseits wurde eine modulare Syntheseroute entwickelt, die neben Calotropin auch weitere natürlich vorkommende und artifizielle Derivate lieferte (Abb. 2, rechts). Beispielsweise wurden verschiedene Strukturmotive an die Position C-17 des Steroidgrundgerüstes [13, 14] angebracht, woraus sich neben der Diversifizierung der Leitstruktur auch neue Bioliganden für Ruthenium-(II)- und Iridium-(III)-Komplexe ergaben [15]. Durch das Molekülarchiv wurden diese katalogisiert und wiederum Kooperationspartnern (Prof. Dr. Ute Schepers, IFG, KIT) für biologische Tests zur Verfügung gestellt.

\section{Das elektronische Laborjournal}

Die Forschung am KIT wird durch ein elektronisches Laborjournal (ELN) und ein Repositorium für Forschungsdaten in vielerlei Hinsicht unterstützt: Insbesondere bei langfristigen und aufwendigen Projekten, die z. B. aus mehreren Teilprojekten bestehen, ergaben sich früher oftmals Probleme beim Teilen von experimentellen Daten unter Kollegen oder Kooperationspartnern. Die digitale Speicherung der Daten ermöglicht es nun den Chemikern, untereinander Wissen auszutauschen. Im Falle des Calotropin-Projektes war es z. B. hilfreich, Daten unter den
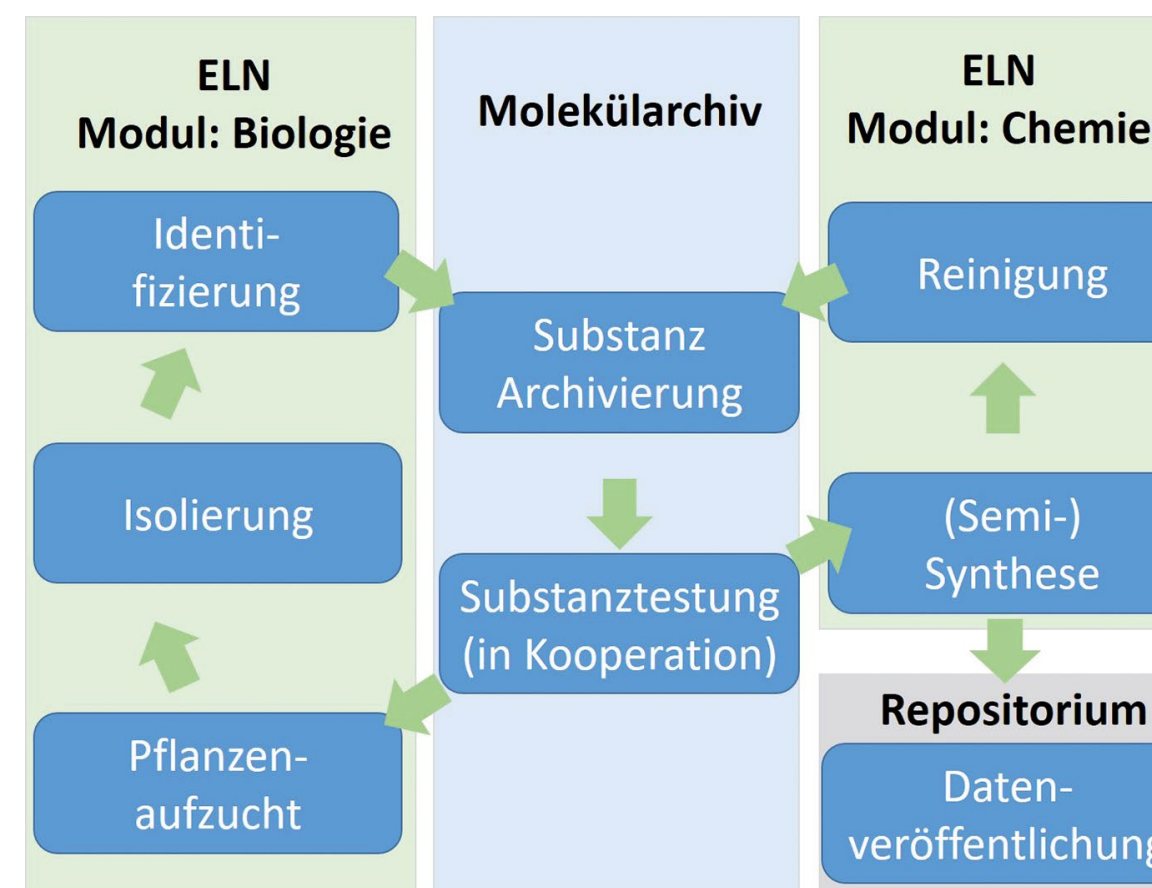

A Abb. 2: Zusammenwirken von Molekülarchiv, elektronischem Laborjournal (ELN) und Repositorium im Rahmen eines Naturstoffprojektes, an dem verschiedene Forschergruppen (Prof. Dr. Stefan Bräse, Prof. Dr. Ute Schepers, Prof. Dr. Peter Nick, Dr. Andrew Cheung) beteiligt sind.

Gruppen, die entweder die Isolation oder die biologischen Tests übernahmen, auszutauschen und sich über den Forschungsfortschritt zu informieren. Dies geschieht im ELN durch die verschiedenen Export- und Importfunktionen sowie die Möglichkeit, ausgewählte Datensätze zu synchronisieren oder zu teilen. Dabei lassen sich individuell Freigabeoptionen mit unterschiedlichen Nutzungsrechten auswählen. Das ELN unterstützt insbesondere die Übergabe von Projekten unter den Wissenschaftlern, wodurch der Verlust von Information vermieden werden kann.

Das ELN ist eine Webanwendung und steht als Open Source zur Verfügung. So können der freie Zugang, eine kostenlose und einfache Nutzung sowie die Möglichkeit zum Modifizieren der Software entsprechend den Anforderungen des jeweiligen Nutzers garantiert werden. Chemiker können ihre Versuche mit dem ELN entwerfen, diese dokumentieren und auch nach Durchführung des Experiments durch die Abspeicherung von aufgenommen analytischen Rohdaten sowie deren Anzeige und Auswertung alle nötigen analytischen Daten hinterlegen. So können beispielsweise NMR(nuclear magnetic resonance)-Spektren, Infrarot(IR)-Spektren und Massenspektren, welche zur eindeutigen Charakterisierung einer chemischen Verbindung nötig sind, gespeichert und so zu jedem Zeitpunkt nachvollzogen werden. Während der Planungsphase kann der Experimentator beispielsweise durch ein SciFinder-Plug-in unterstützt werden, welches die Abfrage von Informationen aus kommerziellen Daten bei vorhandener Lizenz ermöglicht.

\section{Das Repositorium}

Sind alle Daten im ELN zusammengetragen worden, so lassen sich die entsprechenden Forschungsdaten durch die integrierte Schnittstelle in das Repositorium Chemotion transferieren. Dies ermöglicht die unkomplizierte Offenlegung der gewonnenen Daten. Die Bereitstellung der Daten beinhaltet zudem eine parallele DOI-Erstellung für die Forschungsdaten durch DataCite und eine Registrierung der Inhalte in der Strukturdatenbank PubChem.

\section{Typischer Workflow}

Um den Service der Compound Platform zur Molekülarchivierung in Anspruch zu nehmen, ist eine Registrierung bei der Plattform notwendig. Anschließend können die jeweiligen Substanzen in bereitgestellte Glasware abgefüllt werden, sodass ein schneller und sicherer Transport erfolgen kann. Über das elektronische Portal können wichtige Angaben zu der jeweiligen chemischen Verbindung, wie Struktur, Name, Menge oder 
Eigenschaft, abgespeichert werden. Nach einer standardisierten Reinheitsprüfung, übernimmt ComPlat die sachgerechte Lagerung, die digitale Verwaltung der Daten und die Nachnutzung der übermittelten Daten, was in Absprache mit dem zugehörigen Wissenschaftler bzw. der zugehörigen akademischen Institution geschieht. Ebenfalls besteht im Falle eines Kooperationsprojektes, z. B. zur Untersuchung der biologischen Aktivität der Substanzen, die Option, dass ComPlat die Aliquotierung und das Molekülhandling, was das Auswiegen und die Herstellung von Stammlösung umfasst, übernimmt. Nach Versand der Moleküle, verwaltet ComPlat auch die biologischen Ergebnisse und informiert den zugehörigen Chemiker über die erhaltenen Testergebnisse.

\section{Partnerschaft und Kontakt}

Bei Rückfragen zum Chemotion ELN und Repositorium oder Anfragen zur Nutzung der Compound Platform können sich Interessenten und Nutzer über www.compound-platform.eu melden oder sich alternativ direkt an die Korrespondenzadressen wenden. ComPlat sucht stets nach zusätzlichen Partnern aus der organischen Chemie, die bereit sind, ihre Verbindungen zur Verfügung zu stellen, oder nach Partnern aus der Biologie oder pharmazeutischen Chemie, die an einer Nachnutzung interessiert sind.

\section{Danksagung}

Das Chemotion- und ComPlat-Team dankt den Usern und den Kooperationspartnern für ihr Vertrauen in den letzten Jahren und der Deutschen Forschungsgemeinschaft für die finanzielle Unterstützung.

\section{Literatur}

[1] Agrawal AA, Petschenka G, Bingham RA et al. (2012) Toxic cardenolides: chemical ecology and coevolution of specialized plant-herbivore interactions. New Phytol 194:28-45 [2] Bernays EA, Chapman RF (2007) Host-Plant Selection by Phytophagous Insects. Springer US, New York

[3] Petschenka G, Fei CS, Araya JJ et al. (2018) Relative selectivity of plant cardenolides for $\mathrm{Na}^{+} / \mathrm{K}+-\mathrm{ATP}$ ases from th monarch butterfly and non-resistant insects. Front Plant Sci 9:1424

[4] You H, Lei M, Song W et al. (2013) Cytotoxic cardenolides from the root bark of Calotropis gigantea. Steroids 78:10291034

[5] Kupchan SM, Knox JR, Kelsey JE et al. (1994) Calotropin, a cytotoxic principle isolated from Asclepias curassavica L Science 146:1685-1686

[6] Seiber JN, Nelson CJ, Lee SM (1982) Cardenolides in the latex and leaves of seven Asclepias species and Calotropis procera. Phytochemistry 21:2343-2348

[7] Jung N, Deckers A, Bräse S (2017) Ein Molekülarchiv als akademisch integrierte Service-Einrichtung. BIOspektrum 23:212-214

[8] Tremouilhac P, Nguyen A, Huang Y-C et al. (2017)

Chemotion ELN: an Open Source electronic lab notebook for chemists in academia. J Cheminformatics 9:54

[9] Chemotion - Repository for molecules, reactions and research data, https://www.chemotion-repository.net/welcome

[10] Cheung HTA, Chiu FCK, Watson TR et al. (1983)

Cardenolide glycosides of the Asclepiadaceae. New glycosides from Asclepias fruticosa and the stereochemistry of uscharin, voruscharin and calotoxin. J Chem Soc, Perkin Trans 1:28272835

[11] Cheung HTA, Nelson CJ (1989) Cardenolide glycosides with 5,6-unsaturation from Asclepias vestita. J Chem Soc, Perkin Trans 1:1563-1570

[12] Cheung HTA, Nelson CJ, Watson TR (1988) New glucoside conjugates and other cardenolide glycosides from the monarch butterfly reared on Asclepias fruticosa $\mathrm{L}$. J Chem Soc, Perkin Trans 1:1851-1857

[13] Koch V, Bräse S (2017) Pd-mediated cross-coupling of C-17 lithiated androst-16-en-3-ol - access to functionalized arylated steroid derivatives. Org Biomol Chem 15:92-95 [14] Koch V, Nieger M, Bräse S (2017) Stille and Suzuki cross-coupling reactions as versatile tools for modifications at C-17 of steroidal skeletons - a comprehensive study. Adv Synth Catal 359:832-840
[15] Koch V, Meschkov A, Feuerstein W et al. (2019) Synthesis, characterization, and biological properties of steroidal ruthenium(II) and iridium(III) complexes based on the androst-16-en-3-ol framework. Inorg Chem 58:15917-15926 [16] Ishibashi M (2019) Screening for natural products that affect Wnt signaling activity. J Nat Med 73:697-705

[17] Park HY, Toume K, Arai MA et al. (2014) Calotropin: a cardenolide from Calotropis gigantea that inhibits Wnt signaling by increasing casein kinase $1 \alpha$ in colon cancer cells. ChemBioChem 15:872-878

Funding: Open Access funding provided by Projekt DEAL.

Open Access: Dieser Artikel wird unter der Creative Commons Namensnennun 4.0 International Lizenz veröffentlicht, welche die Nutzung, Vervielfältigung, Bearbeitung, Verbreitung und Wiedergabe in jeglichem Medium und Form erlaubt, sofern Sie den/die ursprünglichen Autor(en) und die Quelle
ordnungsgemäß nennen, einen Link zur Creative Commons Lizenz beifügen und ordnungsgemäß nennen, einen Link zur Creative Commons Lizenz beift enthaltenen Bilder und sonstiges Drittmaterial unterliegen ebenfalls der genannten Creative Commons Lizenz, sofern sich aus der Abbildungslegende nichts anderes ergibt. Sofern das betreffende Material nicht unter der genannten Creative Commons Lizenz steht und die betreffende Handlung nicht nach gesetzlichen Vorschriften erlaubt ist, ist für die oben aufgeführten Weiterverwendungen des Materials die Einwilligung des jeweiligen Rechteinhabers einzuholen. Weitere Details zur Lizenz entnehmen Sie bitte der tion uf http://creativecommons.org/licenses/by/4.0/deed.de.

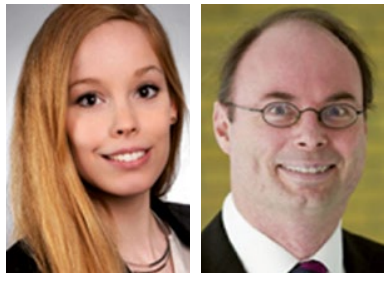

Vanessa Koch und Stefan Bräse

Korrespondenzadresse:

Prof. Dr. Stefan Bräse

Institut für Biologische und Chemische Systeme IBCS-FMS

Karlsruher Institut für Technologie (KIT), Campus Nord

Hermann-von-Helmholtz-Platz 1

D-76344 Eggenstein-Leopoldshafen

braese@kit.edu 